Check for updates

Cite this: Mater. Chem. Front. 2020, 4, 3585

Received 29th April 2020

Accepted 10th June 2020

DOI: 10.1039/d0qm00278j

rsc.li/frontiers-materials

\title{
Protonic acid doping of low band-gap conjugated polyions $\dagger$
}

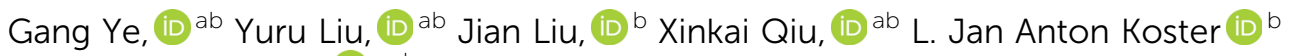 \\ and Ryan C. Chiechi $\mathbb{D}$ *ab
}

\begin{abstract}
This paper describes the design and synthesis of a series of conjugated polyions (CPIZ-T, CPIZ-TT and CPIZ-TT-DEG) that incorporate a formal positive charge into their conjugated backbones, balanced by anionic pendant groups with increasing electron-donating ability. The energy levels and the bandgap of these conjugated polyions were determined by using optical absorption spectroscopy and cyclic voltammetry (CV) and were easily modulated by varying the electron donating group. The energies of the occupied states increase with increasing electron-donating ability, while the energies of the unoccupied states are almost unchanged due to the presence of tritylium ions in the conjugated backbone. All conjugated polyions exhibit pristine semiconducting properties in weak protonic acids, but with sufficiently strong acids, the polymers exhibit spontaneous spin unpairing and convert to a metallic state. The required strength of the acids varies with the electron-donating ability, with higher HOMO levels leading to more facile proton acid doping and higher electrical conductivities. The mechanism of protonic acid doping of conjugated polyions involves a spinless doping process (dehydration) followed by a spontaneous spin unpairing leading to the formation of polarons. While protonic acid doping occurs in polyaniline, conjugated polyions offer synthetic tunability and selective processing into insulating, semiconducting and metallic states simply by controlling acidity.
\end{abstract}

\section{Introduction}

Conjugated polymers combine the utility of organic synthesis and the intrinsic properties of soft materials with the utility of semiconductors. However, the best-performing conjugated polymers tend to require processing from solvents, which is difficult or expensive to scale up. Conjugated polyelectrolytes (CPEs) address this problem by decorating the $\pi$-conjugated backbone with pendant ionic functionalities, enabling processing from polar, protic solvents such as water or ethanol. Although they retain their semiconducting properties, the mismatch in polarity between the backbone and the pendant groups tends to drive unfavorable morphologies for bulk chargetransport. ${ }^{1}$ We have taken a different approach, installing closedshell charges into the $\pi$-conjugated backbone to balance the charged pendant groups, forming conjugated polyions (CPIs). ${ }^{2,3}$

Conjugated polymers that can be processed from green solvents, which tend to be polar and protic, have played a key

\footnotetext{
${ }^{a}$ Stratingh Institute for Chemistry, University of Groningen, Nijenborgh 4,

9747 AG Groningen, The Netherlands. E-mail: r.c.chiechi@rug.nl

${ }^{b}$ Zernike Institute for Advanced Materials, Nijenborgh 4, 9747 AG Groningen,

The Netherlands

$\dagger$ Electronic supplementary information (ESI) available: Detailed description of the synthesis and characterization; details of density functional theory calculations; conductance and Seebeck coefficient measurements. See DOI: 10.1039/d0qm00278j
}

role in a range of applications in organic electronics. ${ }^{4}$ The most widely-used of such polymers is poly(3,4-ethylenedioxythiophene): polystyrene sulfonate (PEDOT:PSS), which is a polymer blend in which the charges of doped PEDOT are balanced by PSS. It is used, for example, as a hole-collection layer in organic solar cells or as an active material in thermoelectric devices. ${ }^{5}$ However, PEDOT:PSS is prepared in the doped, metallic state and is processed from an aqueous suspension. Self-doped PEDOT-based conjugated polyelectrolytes eliminate the need to blend PEDOT with a separate polyelectrolyte and can be used as hole-collection layers in organic solar cells or as an active material in organic electrochemical transistors. ${ }^{6-10}$ These and other CPEs are semiconducting polymers defined by $\pi$-conjugated backbones with pendant ionic functionalities. ${ }^{4,11-15}$ They are primarily used as interfacial modifiers in optoelectronic devices because of the aforementioned morphology issues. ${ }^{4,11,16}$ We are developing a new type of conjugated polymer that combines the beneficial properties of CPEs and PEDOT:PSS. By introducing formal, closed-shell charges into the backbones of conjugated polymers through a process we call "spinless doping", we can combine charged backbones with ionic pendant groups to avoid unfavorable aggregation while preserving the semiconducting properties of the backbone. ${ }^{1,3,17,18}$ As such, they can be processed from highly polar, protonic solvents to give smooth, semiconducting thinfilms that serve as active layers in photovoltaic devices. ${ }^{2}$ 
The donor-acceptor strategy is a powerful tool to tune the bandgap and energy levels of conventional conjugated polymers. Through the delicate modification and combination of different strengths of donor or acceptor building blocks, many polymeric materials have been designed and synthesized for applications in organic electronics. ${ }^{19,20}$ When applied to CPEs, donor-acceptor backbone engineering can cause pristine CPES to become self-doped, in which the ionic pendant groups stabilize the polarons in the backbone. ${ }^{21-25}$ Although CPIs bear charges in both the pendant groups and backbone, no evidence of self-doping has been observed to date; they have only been observed in the insulating and semiconducting forms.

Here, we apply the donor-acceptor strategy to the synthesis of a series of conjugated polymers that undergo spinless doping by protonic acids to generate CPIs. We find that the bandgap and energy levels of CPIs are easily modulated by increasing the strength of the donor building block and that, surprisingly, the resulting CPIs can be further doped by protonic acids effecting conversion to the metallic state. While the aforementioned PEDOT-based CPEs become self-doped by the proximity of the ionic pendant groups to the neutral backbone, ${ }^{26}$ CPIs are zwitterionic and the pendant groups balance the closed-shell cations already present in the backbones even in the pristine, semiconducting state. Optical absorption, electron paramagnetic resonance spectroscopy and electrical conductivity measurements reveal that this spontaneous spin-unpairing occurs more easily in CPIs with higher-lying occupied states, suggesting an internal redox process following the initial spinless doping.

\section{Results and discussion}

\section{Synthesis and characterization}

Fig. 1 shows the synthetic route and the corresponding chemical structures of three CPIs, CPIZ-T, CPIZ-TT and CPIZ-TT-DEG.
The monomers were synthesized according to procedures found in the literature, ${ }^{27-31}$ which are described in detail in the ESI. $\dagger$ To use the donor-acceptor strategy for controlling the bandgap $\left(E_{\mathrm{g}}\right)$ and balancing the cationic and anionic groups, we employed a three-component, palladium-catalyzed Stille polymerization in DMF. Monomer 1 provides the anionic pendant groups in the form of carboxylic acids, Monomer 2 is a carbocation precursor and Monomer 3 is an aromatic distannyl compound with increasing electron-donating units, depicted as Ar, that differentiates the three CPIs. The polymers are prepared in their neutral, insulating forms by refluxing the mixture of monomers overnight. As synthesized, all three polymers are insoluble in non-polar solvents and only sparingly soluble in polar solvents, allowing the removal of impurities and lowmolecular-weight fractions by dialysis in water.

\section{Photophysical properties}

The UV-vis absorption spectra for polymers of CPIZ-T, CPIZ-TT and CPIZ-TT-DEG before and after spinless doping are shown in Fig. 2. The spectra of CPIZ-T, CPIZ-TT and CPIZ-TT-DEG exhibit one, main $\pi-\pi^{*}$ transition, located from $350 \mathrm{~nm}$ to $500 \mathrm{~nm}$, reflecting a decrease in the band-gap $\left(E_{\mathrm{g}}\right)$ with increasing donor strength. Since spinless doping is effected by the generation of closed-shell cations from the loss of $\mathrm{H}_{2} \mathrm{O}$, the degree of doping scales with the strength of the acid from which the CPI is processed. In solution, this process is in equilibrium, with weakly acidic solutions like $\mathrm{CH}_{3} \mathrm{COOH}\left(\mathrm{p} K_{\mathrm{a}}=4.76\right)$ disfavoring the dehydrated, cationic form such that the degree of conjugation, which is defined by the average number of consecutive monomers in the cationic form, scales with acidity. The commensurate decrease in $E_{\mathrm{g}}$ also scales with the strength of the donor because of the emergence of a charge-transfer band that forms because of the acceptor nature of tritylium. This relationship can be seen in Fig. 2b; in acidic solutions, charge-transfer bands appear that follow a trend of decreasing energy with increasing donor

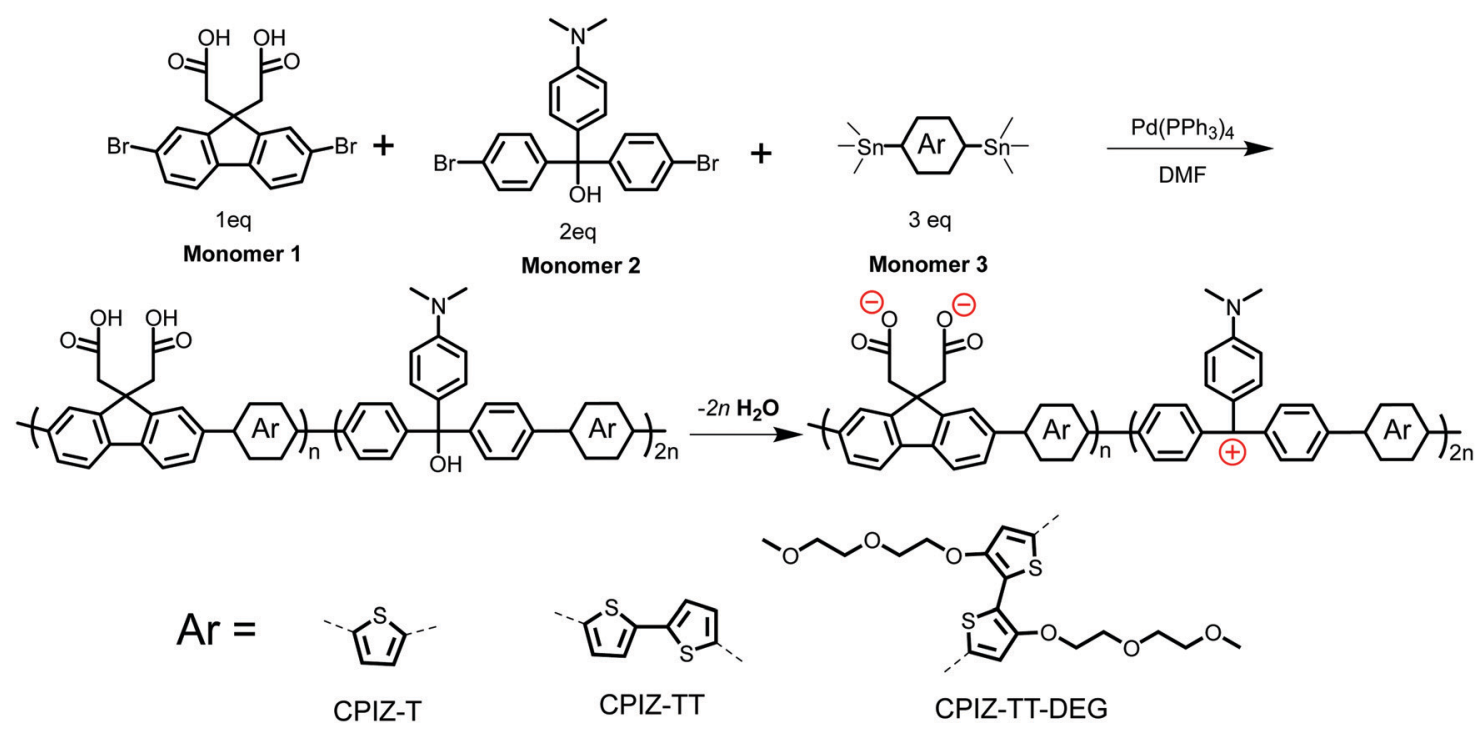

Fig. 1 Synthesis of conjugated polyions via a three-component, random Stille copolymerization. The zwitterionic form is favored in the solid-state by the loss of $\mathrm{H}_{2} \mathrm{O}$. 

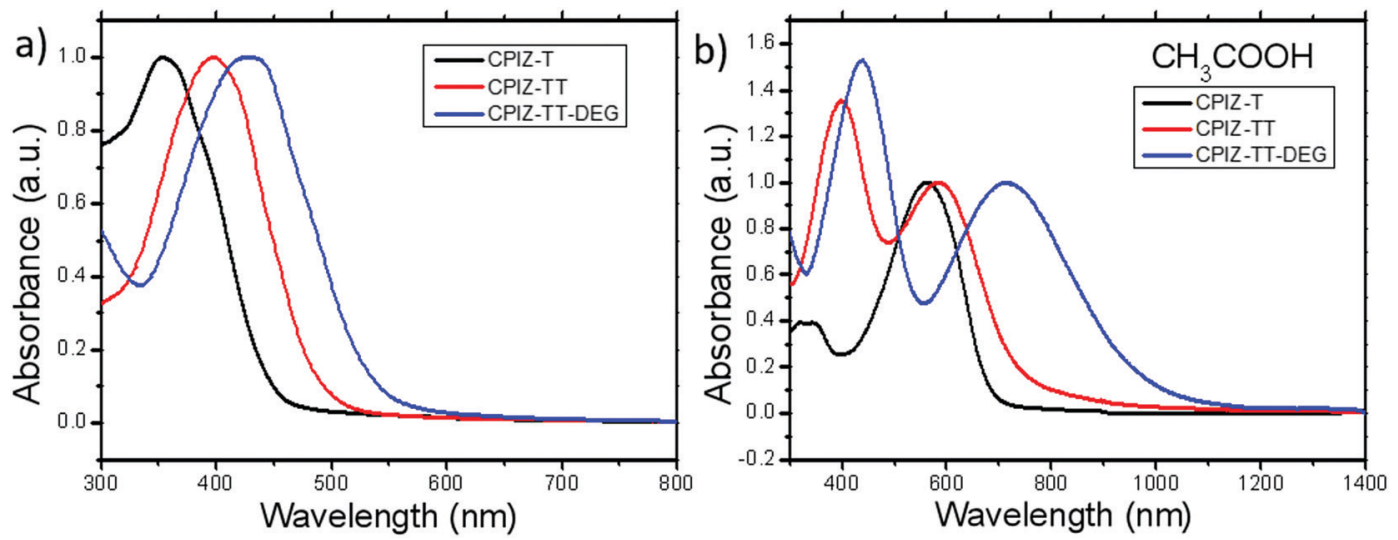

Fig. 2 (a) Absorption spectra of the as-prepared conjugated polyions, CPIZ-T, CPIZ-TT, and CPIZ-TT-DEG, dissolved in either slightly basic $\mathrm{H}_{2} \mathrm{O}$ or DMF $\left(10^{-5} \mathrm{M}\right)$ solutions, showing a decrease in the optical gap with increasing donor strength. (b) Absorption spectra of the same conjugated polyions in glacial $\mathrm{CH}_{3} \mathrm{COOH}\left(10^{-5} \mathrm{M}\right)$, showing a large bathochromic shift associated with the formation of cations in the backbone, which induce a transition to the semiconducting form and increase the conjugation length.

strength. Based on the absorption band edge, the $E_{\mathrm{g}}$ of the CPIs in the cationic form are $1.82 \mathrm{eV}$ for CPIZ-T, $1.66 \mathrm{eV}$ for CPIZ-TT, and $1.29 \mathrm{eV}$ for CPIZ-TT-DEG.

Fig. 3a shows the absorption spectra of CPIZ-T, CPIZ-TT and CPIZ-TT-DEG dissolved in the strong acid $\mathrm{HCOOH}\left(\mathrm{p} K_{\mathrm{a}}=3.77\right)$. As discussed above, the expectation is that the conjugation lengths would increase compared to $\mathrm{CH}_{3} \mathrm{COOH}$, following the same trend in donor-strengths; however, an unexpected, lowenergy transition at $1200 \mathrm{~nm}$ is present in the CPIZ-TT-DEG spectrum that is absent in both the CPIZ-T and CPIZ-TT spectra. Fig. 3b shows that the spectrum of CPIZ-TT-DEG in the even stronger acid $\mathrm{CF}_{3} \mathrm{COOH}\left(\mathrm{p} K_{\mathrm{a}}=-0.25\right)$ exhibits a further bathochromic shift in $\lambda_{\text {max }}$, while the low-energy transition increases in intensity without shifting in energy. The spectra of CPIZ-T and CPIZ-TT show the appearance of a very weak transition at the band edge, revealing a trend with increasing donor-strength.

To compare the effects of increased acidity directly, we re-plotted the absorption spectra of CPIZ-T, CPIZ-TT and CPIZ-TT-DEG separately in the three different acids in Fig. S2 (ESI $\dagger$ ). Although there is a small shoulder in $\mathrm{CF}_{3} \mathrm{COOH}$, the low-energy transition is not present in CPIZ-T in $\mathrm{CH}_{3} \mathrm{COOH}$ or $\mathrm{HCOOH}$. The same trend is apparent in CPIZ-TT, with a slightly higher intensity creating a resolvable transition near $1000 \mathrm{~nm}$. Although these spectra are all in solution, the trends in $\lambda_{\text {max }}$ coupled with the changing intensity of the transition at $1200 \mathrm{~nm}$ (for CPIZ-TT-DEG) are reminiscent of the appearance of polarons in redox-doped conducting polymers.

For further insight into the low-energy transition, we measured the absorption spectra of the thin films of the CPIs cast from different acidic solutions. Fig. S3 (ESI $\dagger$ ) shows the UV-vis-NIR spectra of these films for CPIZ-TT and CPIZ-TT-DEG; the spectra of CPIZ-T are reported in our previous work. ${ }^{2}$ The spectra of CPIZ-T and CPIZ-TT are featureless above $\sim 1000 \mathrm{~nm}$, indicating the absence of polarons or bipolarons. The spectra of the thin films of CPIZ-TT-DEG cast from $\mathrm{CH}_{3} \mathrm{COOH}$ are also featureless above $\sim 1000 \mathrm{~nm}$; however, when cast from either $\mathrm{HCOOH}$ or $\mathrm{CH}_{3} \mathrm{COOH}$, a broad absorption past $1000 \mathrm{~nm}$ appears, indicating the likely formation of polarons.

Unlike the closed-shell cations that form via spinless doping, polarons comprise both spin and charge; they are delocalized radical cations. Thus, we acquired the electron paramagnetic resonance (EPR) spectra for CPIZ-T, CPIZ-TT
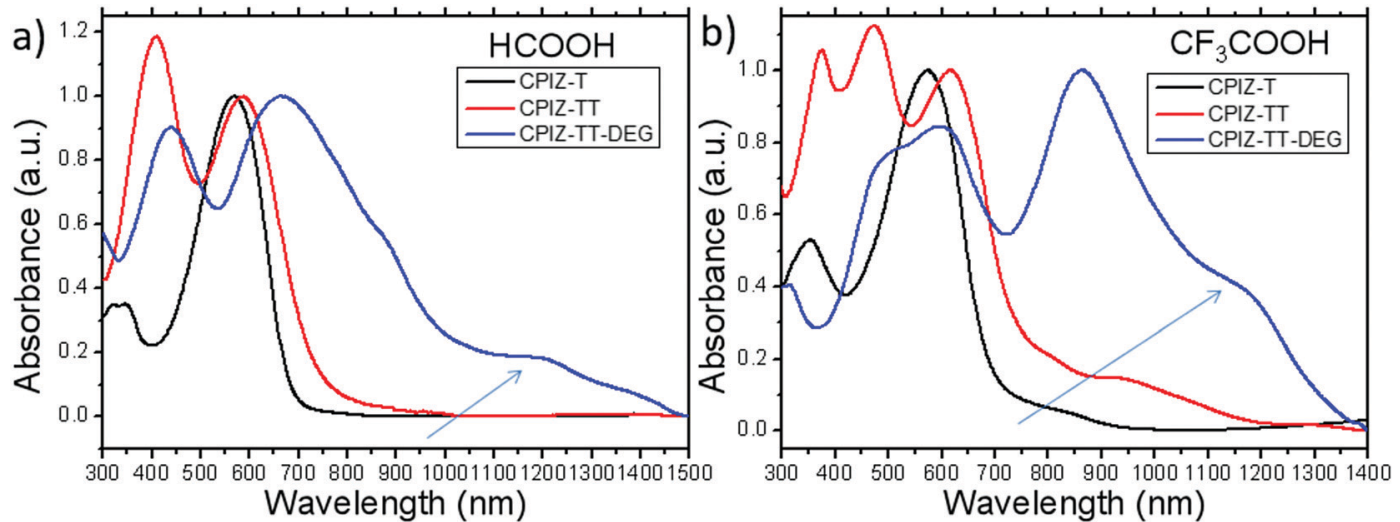

Fig. 3 (a) Absorption spectra of CPIZ-T, CPIZ-TT and CPIZ-TT-DEG in pure $\mathrm{HCOOH}$ corresponding to the formation of delocalized cations. (b) Absorption spectra of CPIZ-T, CPIZ-TT and CPIZ-TT-DEG in pure $\mathrm{CF}_{3} \mathrm{COOH}$. 

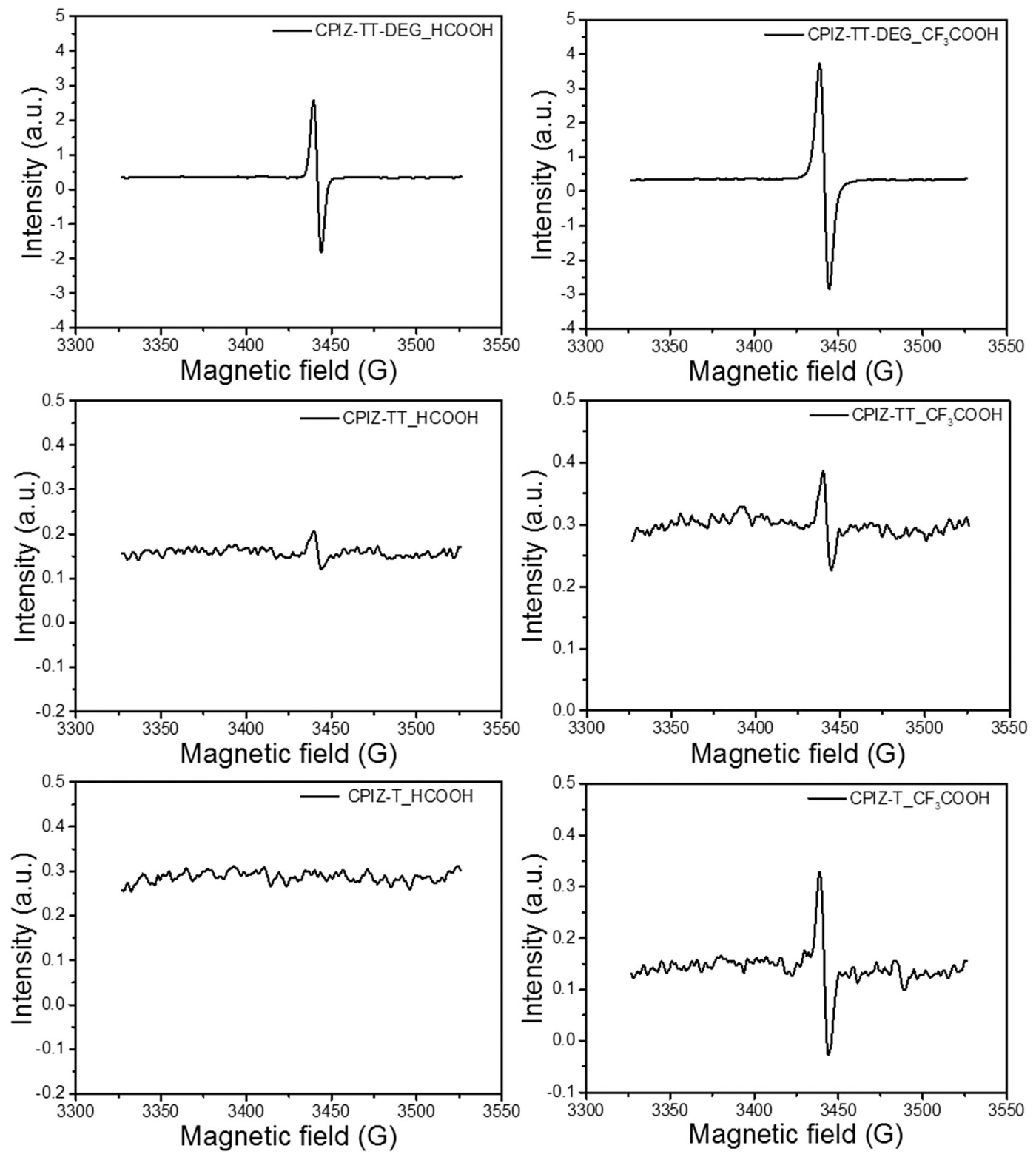

Fig. 4 EPR spectra of solutions of CPIZ-T, CPIZ-TT, and CPIZ-TT in $\mathrm{HCOOH}$ and $\mathrm{CF}_{3} \mathrm{COOH}$.

and CPIZ-TT-DEG in strong acid solutions to investigate the possibility that radical cations form spontaneously in sufficiently strong acids with sufficiently strong donors. The resulting spectra, shown in Fig. 4, reveal that CPIZ-T and CPIZ-TT are EPR-silent in $\mathrm{HCOOH}$, in agreement with the absorption spectra in solution and the solid-state. While, even in $\mathrm{HCOOH}$, CPIZ-TT-DEG shows a strong resonance, confirming that the low-energy transition observed in the absorption spectra corresponds to polarons/radical cations. In $\mathrm{CF}_{3} \mathrm{COOH}$, all three CPIs show a resonance, but it is much stronger in CPIZ-TT-DEG than in CPIZ-TT or CPIZ-T, suggesting that the shoulder in the absorbance spectra corresponds to the formation of very dilute polarons/radical cations. The slightly lower intensity of the EPR signal of CPIZ-TT compared to CPIZ-T contradicts the trend in the absorption intensities, but those may be affected by their aggregation states in $\mathrm{CF}_{3} \mathrm{COOH}$. In any case,
CPIZ-TT-DEG shows the strongest signal intensity by far and the intensity of the EPR signals broadly correlates with the optical band at the NIR regions of the absorption spectra, indicating that these optical transitions are indeed proportional to the concentration of radical species, which is commensurate with the degree of redox doping in the solid-state. The overall trend indicates that the spontaneous formation of polarons correlates to the donorstrength much more significantly than to the strength of the acid from which the films are cast.

\section{Electrochemical properties}

While spinless doping is a two-electron process, eliminating an equivalent of $\mathrm{H}_{2} \mathrm{O}$ to form a closed-shell cation in the backbone and the corresponding pendant carboxylate the formation of unpaired electrons implies the existence of a subsequent redox 
process. Thus, we used cyclic voltammetry (CV) to gain insight into the redox behavior of CPIZ-T, CPIZ-TT, and CPIZ-TT-DEG. The results are shown in Fig. 5; experimental details are provided in the ESI. $\dagger$ All three polymers exhibit fully reversible reduction waves, corresponding to the reduction of tritylium moieties. Although electrochemical reduction normally leads to n-doping, in the case of CPIs, it forms positive polarons (by reducing closed-shell cations) and is, therefore, p-doping. All three polymers exhibit irreversible oxidation curves because CPIs in their semiconducting form are already fully cationic such that the removal of additional electrons corresponds to over-oxidation in traditional conjugated polymers, however, CPIZ-TT-DEG is arguably quasi-reversible. The onset oxidation potentials ( $E_{\text {onset }}^{\text {Ox. }}$ ) of CPIZ-T, CPIZ-TT, and CPIZ-TT-DEG are $0.86,0.68$, and $-0.1 \mathrm{~V}$, respectively. The energy levels of the polymers calculated from these data are summarized in Table 1. Both the occupied (HOMO/valence band) and unoccupied (LUMO/conduction band) levels of the polymers increase gradually with increasing donor-strength; however, the unoccupied states show less sensitivity than the occupied states, presumably because the effects of the donor are swamped by those of the cationic tritylium units. As predicted from the relative strengths of the donors, CPIZ-TT-DEG exhibits the highest-lying occupied state, making it both more energetically favorable to form cations in acidic solutions and for subsequent oxidation.

\section{Density functional theory calculations}

To gain insight into the electronic structure and donor/acceptor character of CPIZ-T and CPIZ-TT, and CPIZ-TT-DEG, we carried out density functional theory (DFT) calculations at the B3LYP/ $6-311 \mathrm{G}(\mathrm{d}, \mathrm{p})$ level using Gaussian $16 .^{32}$ To simplify the calculations, we considered only one repeat-unit comprising two cations, which is sufficient to reveal any anomalies in the frontier orbitals. As can be seen in Fig. 6, the HOMOs are delocalized over the neutral fluorene and thiophene moieties, while the LUMOs are localized on the cationic tritylium moieties, reflecting their strong acceptor nature. The only variation is in CPIZ-TT-DEG, where the HOMO density clearly extends to
Table 1 Summary of the photophysical properties and electrochemical properties of CPIZ-T, CPIZ-TT, and CPIZ-TT-DEG

\begin{tabular}{llll}
\hline Physical properties & CPIZ-T & CPIZ-TT & CPIZ-TT-DEG \\
\hline$\lambda_{\text {onset }}$ solution $^{a}(\mathrm{~nm})$ & 680 & 745 & 960 \\
$E_{\mathrm{g}}^{\text {opt. }}(\mathrm{eV})$ & 1.82 & 1.66 & 1.29 \\
$E_{\mathrm{g}} \mathrm{CV}(\mathrm{eV})$ & 1.81 & 1.66 & 1.05 \\
$E_{\text {Onset }}^{\text {Ped }}(\mathrm{V})$ & -0.54 & -0.58 & -0.62 \\
$E_{\text {onset }}^{\text {ox }}(\mathrm{V})$ & 0.86 & 0.68 & -0.10 \\
${\text { Conduction band from } \mathrm{CV}^{c}(\mathrm{eV})}$ & -4.54 & -4.48 & -4.44 \\
${\text { Valence band from } \mathrm{CV}^{d}(\mathrm{eV})}$ & -5.92 & -5.74 & -4.96
\end{tabular}

${ }^{a}$ CPIs in $\mathrm{CH}_{3} \mathrm{COOH}$ solution. ${ }^{b} E_{\mathrm{g}}^{\text {opt. }}=1240 / \lambda_{\text {onset. }}{ }^{c}$ Calculated from $\mathrm{CV}: E_{\text {conduction }}=-\left(5.06+E_{\text {onset }}^{\text {red }}\right) \mathrm{eV} .{ }^{d}$ Calculated from CV: $E_{\text {valence }}=$ $-\left(5.06+E_{\text {onset }}^{\text {ox. }}\right)$ eV.

the cationic tritylium moieties, leading to some spacial overlap of the frontier orbitals. These results show that the electronic structure of the CPIs does not differ significantly from that of a typical donor-acceptor polymer, despite the presence of cations in the backbone. They also suggest that the donor and acceptor units are the most strongly coupled in CPIZ-TT-DEG, which is reflected in the small bandgap (1.05 eV electrochemically, $1.29 \mathrm{eV}$ optically).

\section{Conductivity}

Redox doping, whether chemical or electrochemical, causes a transition in conjugated polymers from the semiconducting state to the metallic state, which is defined by the presence of free charge carriers (i.e., polarons). ${ }^{33}$ This property is vital to their performance when either high conductivity or carrier density is required. Thus, changes in conductivity are an indirect measure of the formation of free carriers and the transition to a metallic state. To probe the differences in conductivity across the series of CPIs, we used a two-point probe to measure the current-voltage response of thin films of CPIZ-TT and CPIZ-TT-DEG that were spin cast from $\mathrm{HCOOH}$ or $\mathrm{CF}_{3} \mathrm{COOH}$. CPIZ-TT was spin-coated from $5 \mathrm{mg} \mathrm{mL} \mathrm{mL}^{-1}$ in $\mathrm{HCOOH}$ or $\mathrm{CF}_{3} \mathrm{COOH}$, while CPIZ-TT-DEG was spin-coated from $10 \mathrm{mg} \mathrm{mL}{ }^{-1}$ in $\mathrm{HCOOH}$ or $\mathrm{CF}_{3} \mathrm{COOH}$. The results are shown in Fig. 7 . The highest recorded conductivity was
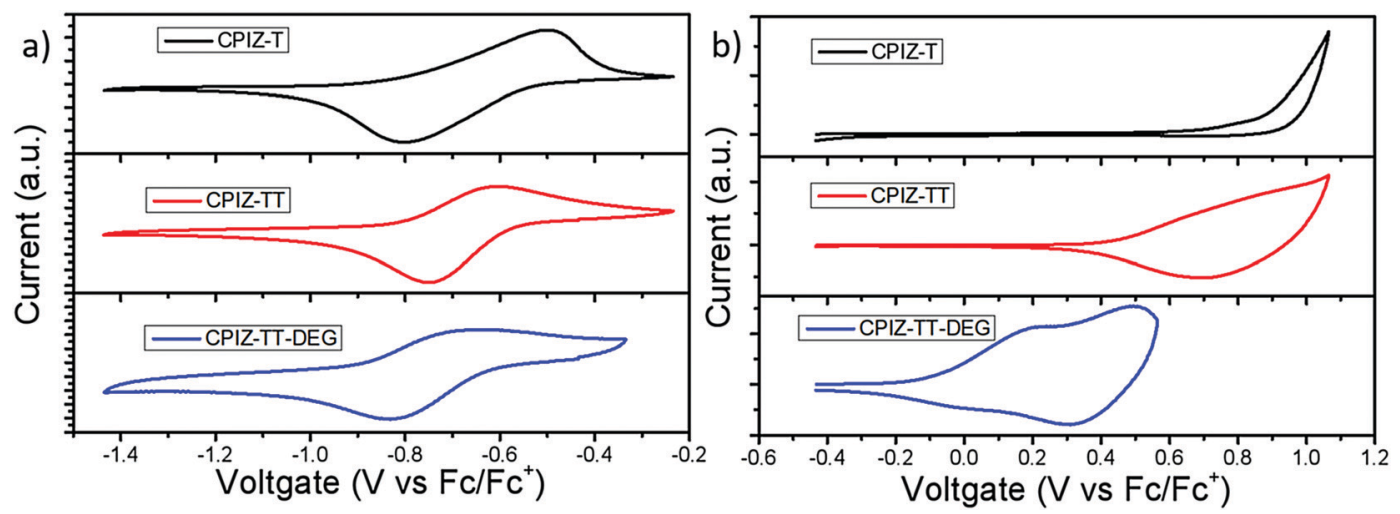

Fig. 5 Cyclic voltammograms of a thin film of CPIZ-T, CPIZ-TT, and CPIZ-TT-DEG versus Fc/Fc ${ }^{+}$on a glassy carbon working electrode immersed in $0.1 \mathrm{~mol} \mathrm{~L}{ }^{-1} n-\mathrm{Bu}_{4} \mathrm{NPF}_{6}$ acetonitrile solution at $100 \mathrm{mV} \mathrm{s}^{-1}$. (a) The fully reversible reduction waves are indicative of the traditional redox doping/ de-doping of the band structure of a semiconducting state conjugated polymer. (b) Oxidative waves are irreversible. 
HOMO
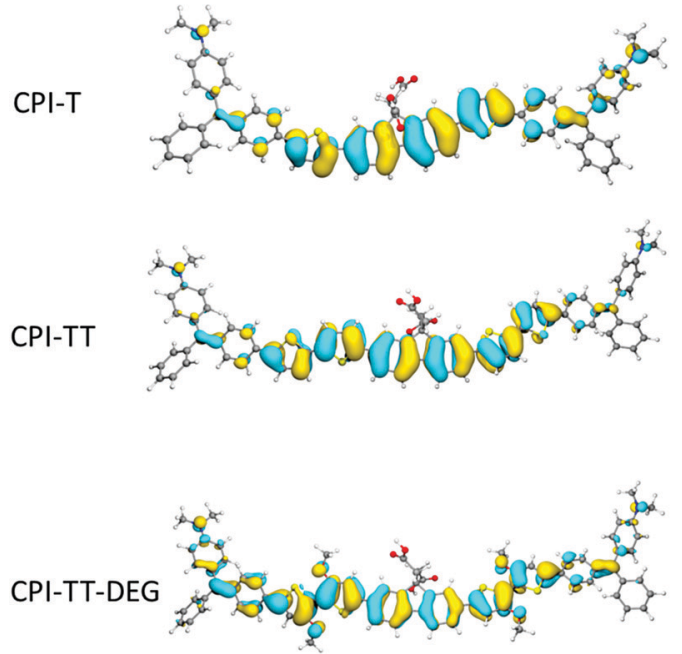

LUMO
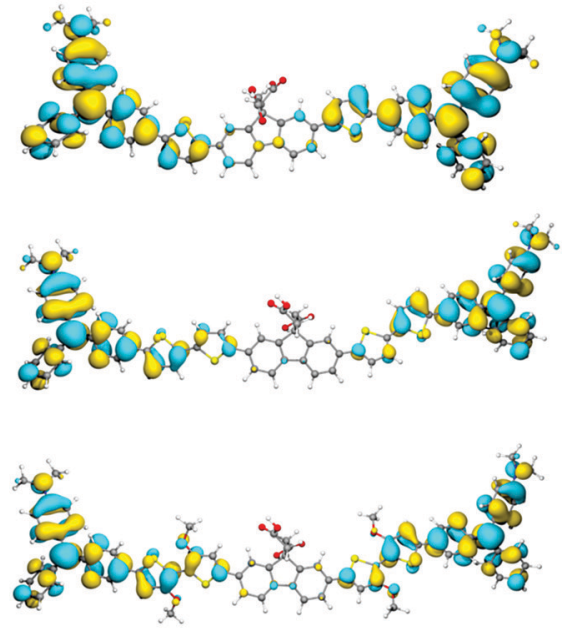

Fig. 6 DFT-optimized molecular orbitals of CPIZ-T, CPIZ-TT, and CPIZ-TT-DEG.

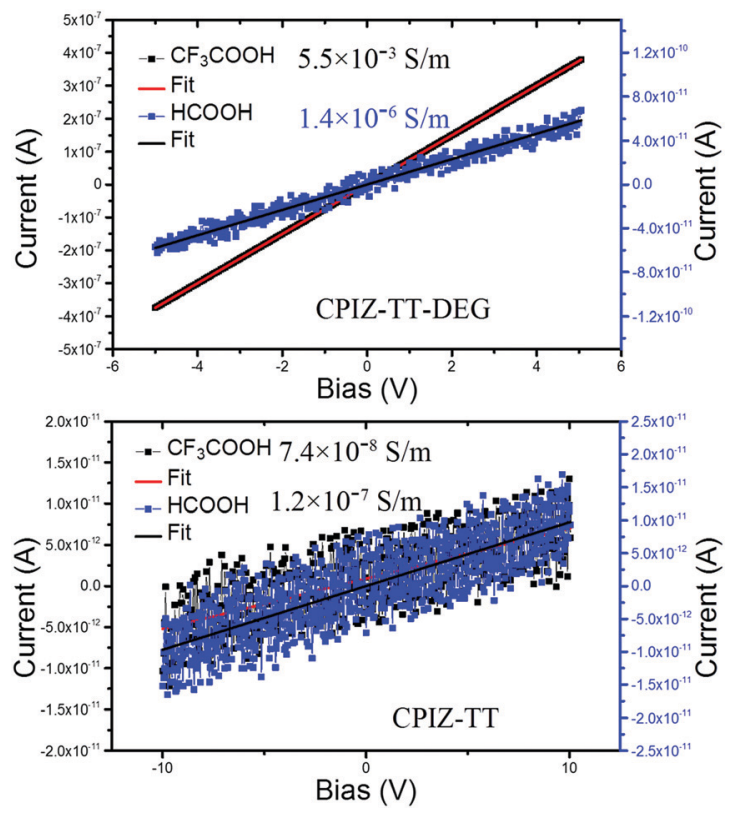

Fig. 7 Current-voltage plots of thin films of CPIZ-TT and CPIZ-TT-DEG cast from $\mathrm{HCOOH}$ and $\mathrm{CF}_{3} \mathrm{COOH}$.

$5.5 \times 10^{-3} \mathrm{~S} \mathrm{~m}^{-1}$ in CPIZ-TT-DEG cast from $\mathrm{CF}_{3} \mathrm{COOH}$, which is four orders of magnitude higher than that of CPIZ-TT. (Device channel-lengths had a negligible effect, as shown in Fig. S5, ESI. $\dagger$ ) The conductivity of the films of CPIZ-TT-DEG cast from $\mathrm{CF}_{3} \mathrm{COOH}$ was three orders of magnitude higher than those cast from $\mathrm{HCOOH}$, while the difference between the films of CPIZ-TT cast from $\mathrm{CF}_{3} \mathrm{COOH}$ and $\mathrm{HCOOH}$ was negligible. This difference could reflect a non-linear relationship between donor-strength and protonic acid-induced doping or a difference in aggregation, but in either case the conductivities of the thin films correlate well to the EPR signal-strengths in solution. The conductivity and carrier mobility of CPIZ-T are reported in our previous work; as expected, it is the least conductive of the series. $^{2}$

\section{Film morphology}

The putative advantage of CPIs, in general, over traditional conjugated polymers, and especially CPEs, is that they are directly solubilized by polar, protonic solvents rather than forming aggregates or suspensions; their zwitterionic nature, in which opposing charges exist in the backbone and pendant groups, means that the polymer chains are completely solvated, leading to smooth, homogeneous films. Fig. S4 (ESI $\dagger$ ) shows the height images of CPIZ-TT-DEG films on bare glass substrates measured by using atomic force microscopy (AFM). Films cast from $10 \mathrm{mg} \mathrm{mL}{ }^{-1}$ in $\mathrm{HCOOH}$ are smoother than those from $10 \mathrm{mg} \mathrm{mL}^{-1}$ in $\mathrm{CF}_{3} \mathrm{COOH}$ with the RMS roughness values of $0.56 \mathrm{~nm}$ and $2.44 \mathrm{~nm}$, respectively.

\section{Seebeck coefficient}

The low thermal conductivity of conjugated polymers makes them ideally suited for use in organic thermoelectric devices; but since electric currents are driven by the migration of carriers along a thermal gradient, they must be used in their doped form. One possible application of doped CPIs is as p-type thermoelectric materials that can be cast from green solvents and that do not require any additional doping. In order to test the viability of the doped CPIZ-TT-DEG and study the polarity of the doping, we determined the Seebeck coefficient of the films cast from $\mathrm{CF}_{3} \mathrm{COOH}$ to be $111 \mu \mathrm{V} \mathrm{K}^{-1}$, from the data shown in Fig. S6 (ESI $\dagger$ ). Using the electrical conductivity and Seebeck coefficient, we also calculated the power factors of the films of CPIZ-TT-DEG. Films cast from $\mathrm{CF}_{3} \mathrm{COOH}$ give a power factor of $6.8 \times 10^{-5} \mu \mathrm{W} \mathrm{m} \mathrm{m}^{-1} \mathrm{~K}^{-2}$. The positive sign of the Seebeck coefficient further indicates that the doped CPIZ-TT-DEG is a p-type polymer, in agreement with the hypothesis that positive polarons form spontaneously from the closed-shell cations. 

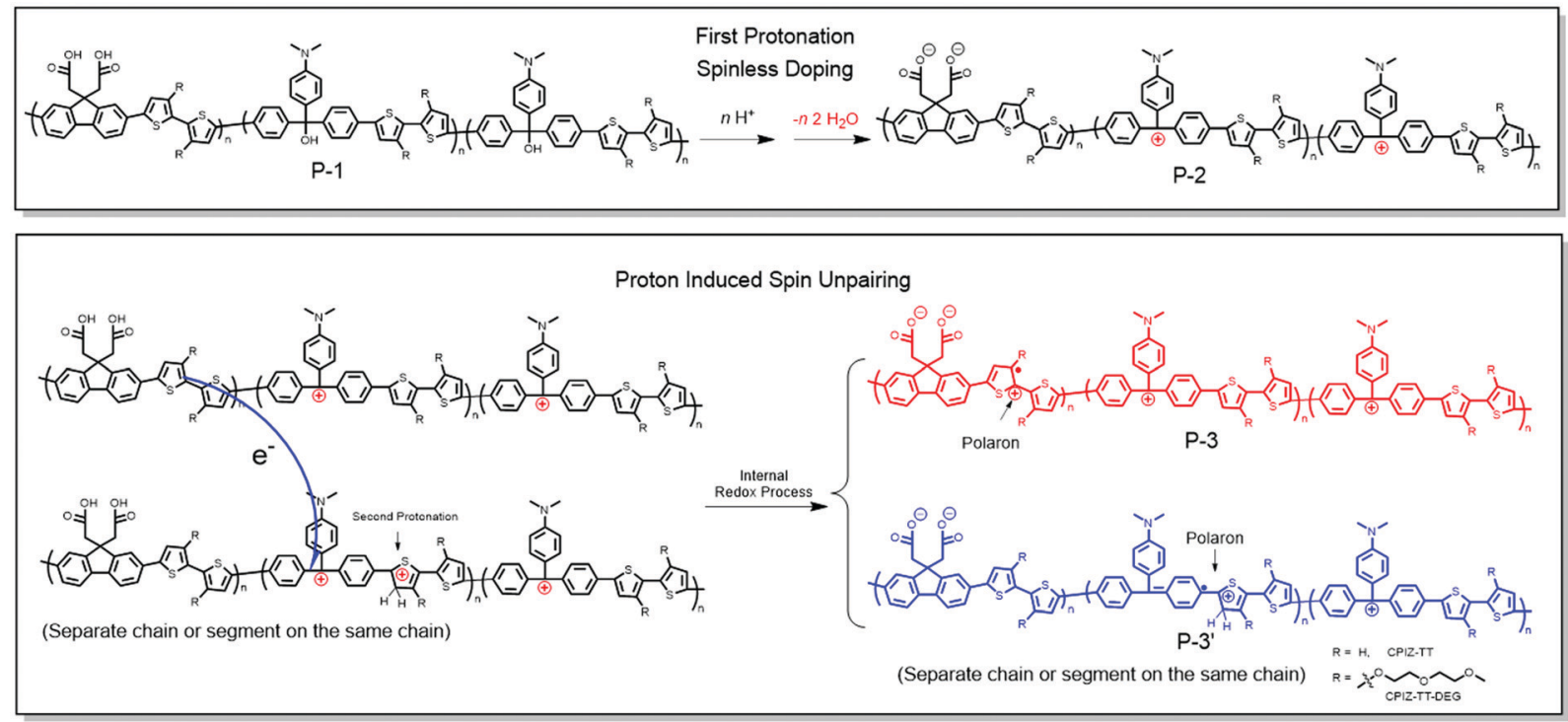

Fig. 8 Proposed proton-induced spin unpairing mechanism (PISUM) for conjugated polyions (CPIs). The protonation is assumed to take place on a thiophene ring, but the exact position is uncertain.

\section{Proton-induced spin unpairing}

The exact mechanism by which CPIs form polarons from protonic acid doping cannot be determined unambiguously, but we can formulate a plausible explanation from the experimental data described above. Protonic acids as non-oxidizing dopants for conventional conjugated polymers such as poly(phenylenevinylene) and polyaniline have been systematically investigated. ${ }^{34}$ But the mechanism for the proton-induced spin unpairing mechanism (PISUM) was first proposed by Wudl et al. in $1987 .{ }^{35}$ In principle, the PISUM in CPIs is no different than for the ordinary conjugated polymers, since the CPIs are pristine semiconductors, even though they bear charges. We observed that the PISUM of CPIs is such that, the lower the energy of the occupied states, the stronger the acid required to induce a signal by EPR. This trend is consistent with the conventional conjugated polymers for which protonic acid doping scales with ionization potential. ${ }^{34}$ Therefore, we proposed the PISUM shown in Fig. 8 based on the results above and PISUM in conventional conjugated polymers. The first step is spinless doping, in which protonation converts an insulating CPI precursor (P-1) into a pristine semiconductor $(\mathrm{P}-2) .^{1-3,17}$ This step is always exergonic and becomes more favorable with increasing donor-strength (Table $\mathrm{S} 1, \mathrm{ESI} \dagger$ ). There is a redox imbalance between the donor and acceptor units, but this is true for all donor/acceptor copolymers; for homopolymers like PANI, this redox imbalance develops when part of a polymer chain is protonated, but in the case of CPIs, the first protonation effects spinless doping, before which the polymer backbone is not conjugated. Thus, we propose that the second protonation of the donor unit of one polymer chain widens the redox imbalance sufficiently to drive the redox reaction and an electron transfer from one P-2 chain to another protonated P-2 chain (or another segment of the same chain), resulting in the formation of the polaron segments $\mathrm{P}-3$ and P- $3^{\prime}$. According to this mechanism, PISUM in CPIs would become more favorable as the occupied state increases in energy both because it is driven by a redox imbalance between the donor and acceptor (which may also be facilitated by the spatial overlap of the HOMO and LUMO revealed by the DFT calculations) and because it depends on the protonation of the donor moiety, which is more energetically favorable in more electron-rich donors.

\section{Conclusion}

Our previous studies on CPIs have focused on their semiconducting properties and the ability to cast them into smooth, homogeneous films from green solvents. The serendipitous discovery that CPIZ-TT-DEG spontaneously forms polarons in acidic solutions extends the utility of CPIs to applications that require doped polymers/free charge-carriers. The catonic nature of CPIs is such that, in their semiconducting form, the backbone contains positive charges, yet electrochemical reduction leads to p-doping; because the PISUM in traditional conjugated polymers forms $\mathrm{p}$-doped polymers via oxidation, one would not expect to find it in CPIs. The fact that we observed a PISUM further underscores the otherwise ordinary nature of CPIs; they are intrinsic semiconducting polymers that exhibit a transition to the metallic state upon doping, except that they are completely soluble in polar, protonic solvents and can be deposited in their semiconducting or doped forms simply by controlling the acidity of the solution from which they are processed.

\section{Conflicts of interest}

There are no conflicts to declare. 


\section{Acknowledgements}

This work is part of the research program of the Foundation for Fundamental Research on Matter (FOM), which is part of The Netherlands Organization for Scientific Research (NWO). This is a publication by the FOM Focus Group "Next Generation Organic Photovoltaics", participating in the Dutch Institute for Fundamental Energy. G. Y. and Y. L. acknowledge financial support from the China Scholarship Council (CSC). The work by J. L. is supported by a grant from STW/NWO (VIDI 13476). $\mathrm{X}$. Q. acknowledge financial support from the Zernike Institute of Advanced Materials.

\section{References}

1 T. P. Voortman and R. C. Chiechi, Thin Films Formed From Conjugated Polymers With Ionic, Water-Soluble Backbones, ACS Appl. Mater. Interfaces, 2015, 7, 28006-28012.

2 G. Ye, N. Y. Doumon, S. Rousseva, Y. Liu, M. Abdu-aguye, M. A. Loi, J. C. Hummelen, L. J. A. Koster and R. C. Chiechi, Conjugated Polyions Enable Organic Photovoltaics Processed From Green Solvents, ACS Appl. Energy Mater., 2019, 2, 2197-2204.

3 T. P. Voortman, H. D. de Gier, R. W. A. Havenith and R. C. Chiechi, Stabilizing Cations in the Backbones of Conjugated Polymers, J. Mater. Chem., 2014, 2, 3407-3415.

4 C. Duan, K. Zhang, C. Zhong, F. Huang and Y. Cao, Recent Advances in Water/Alcohol-Soluble P-Conjugated Materials: New Materials and Growing Applications in Solar Cells, Chem. Soc. Rev., 2013, 42, 9071-9104.

5 B. Xu and J. Hou, Solution-Processable Conjugated Polymers as Anode Interfacial Layer Materials for Organic Solar Cells, Adv. Energy Mater., 2018, 1800022.

6 W. Cai, C. Musumeci, F. N. Ajjan, Q. Bao, Z. Ma, Z. Tang and O. Inganäs, Self-doped conjugated polyelectrolyte with tuneable work function for effective hole transport in polymer solar cells, J. Mater. Chem. A, 2016, 4, 15670-15675.

7 C. Musumeci, M. Vagin, E. Zeglio, L. Ouyang, R. Gabrielsson and O. Inganäs, Organic electrochemical transistors from supramolecular complexes of conjugated polyelectrolyte PEDOTS, J. Mater. Chem. C, 2019, 7, 2987-2993.

8 K. M. Persson, R. Gabrielsson, A. Sawatdee, D. Nilsson, P. Konradsson and M. Berggren, Electronic control over detachment of a self-dopedwater-soluble conjugated polyelectrolyte, Langmuir, 2014, 30, 6257-6266.

9 E. Zeglio, M. Vagin, C. Musumeci, F. N. Ajjan, R. Gabrielsson, X. T. Trinh, N. T. Son, A. Maziz, N. Solin and O. Inganäs, Conjugated Polyelectrolyte Blends for Electrochromic and Electrochemical Transistor Devices, Chem. Mater., 2015, 27, 6385-6393.

10 E. Zeglio, J. Eriksson, R. Gabrielsson, N. Solin and O. Inganäs, Highly Stable Conjugated Polyelectrolytes forWater-Based Hybrid Mode Electrochemical Transistors, Adv. Mater., 2017, 29, 6-11.

11 C. C. Chueh, C. Z. Li and A. K. Jen, Recent Progress and Perspective in Solution-Processed Interfacial Materials for
Efficient and Stable Polymer and Organometal Perovskite Solar Cells, Energy Environ. Sci., 2015, 8, 1160-1189.

12 Z. B. Henson, Y. Zhang, T.-Q. Nguyen, J. H. Seo and G. C. Bazan, Synthesis and Properties of Two Cationic Narrow Band Gap Conjugated Polyelectrolytes, J. Am. Chem. Soc., 2013, 135, 4163-4166.

13 J. H. Seo, A. Gutacker, Y. Sun, H. Wu, F. Huang, Y. Cao, U. Scherf, A. J. Heeger and G. C. Bazan, Improved High-Efficiency Organic Solar Cells via Incorporation of a Conjugated Polyelectrolyte Interlayer, J. Am. Chem. Soc., 2011, 133, 8416-8419.

14 W. Lee, J. H. Seo and H. Y. Woo, Conjugated polyelectrolytes: a new class of semiconducting material for organic electronic devices, Polymer, 2013, 54, 5104-5121.

15 J. E. Houston, S. Richeter, S. Clément and R. C. Evans, Molecular design of interfacial layers based on conjugated polythiophenes for polymer and hybrid solar cells, Polym. Int., 2017, 66, 1333-1348.

16 Z. He, B. Xiao, F. Liu, H. Wu, Y. Yang, S. Xiao, C. Wang, T. P. Russell and Y. Cao, Single-Junction Polymer Solar Cells With High Efficiency and Photovoltage, Nat. Photonics, 2015, 9, 174-179.

17 T. P. Voortman, D. Bartesaghi, L. J. A. Koster and R. C. Chiechi, Cross-Conjugated N-Dopable Aromatic Polyketone, Macromolecules, 2015, 48, 7007-7014.

18 R. C. Chiechi, G. Sonmez and F. Wudl, A Robust Electroactive N-Dopable Aromatic Polyketone, Adv. Funct. Mater., 2005, 15, 427-432.

19 L. Dou, Y. Liu, Z. Hong, G. Li and Y. Yang, Low-Bandgap Near-Ir Conjugated Polymers/Molecules for Organic Electronics, Chem. Rev., 2015, 115, 12633-12665.

20 Y. J. Cheng, S. H. Yang and C. S. Hsu, Synthesis of Conjugated Polymers for Organic Solar Cell Applications, Chem. Rev., 2009, 109, 5868-5923.

21 C.-K. Mai, H. Zhou, Y. Zhang, Z. B. Henson, T.-Q. Nguyen, A. J. Heeger and G. C. Bazan, Facile Doping of Anionic Narrow-Band-Gap Conjugated Polyelectro-Lytes During Dialysis, Angew. Chem., Int. Ed., 2013, 52, 12874-12878.

22 C.-K. Mai, R. A. Schlitz, G. M. Su, D. Spitzer, X. Wang, S. L. Fronk, D. G. Cahill, M. L. Chabinyc and G. C. Bazan, Side-Chain Effects on the Conductivity, Morphology, and Thermoelectric Properties of Self-Doped Narrow-Band-Gap Conjugated Polyelectrolytes, J. Am. Chem. Soc., 2014, 136, 13478-13481.

23 C.-K. Mai, T. Arai, X. Liu, S. L. Fronk, G. M. Su, R. A. Segalman, L. Chabinyc and G. C. Bazan, Electrical properties of doped conjugated polyelectrolytes with modulated density of the ionic functionalities, Chem. Commun., 2015, 51, 1-4.

24 D. X. Cao, D. Leifert, V. Brus, M. S. Wong, H. Phan, B. Yurash, N. Koch, G. C. Bazan and T.-Q. Nguyen, The Importance of Sulfonate to the Self-doping Mechanism of the Water-Soluble Conjugated Polyelectrolyte PCPDTBTSO3K, Mater. Chem. Front., 2020, DOI: 10.1039/d0qm00073f.

25 D. Tsokkou, L. Peterhans, D. X. Cao, C. K. Mai, G. C. Bazan, T. Q. Nguyen and N. Banerji, Excited State Dynamics of a 
Self-Doped Conjugated Polyelectrolyte, Adv. Funct. Mater., 2020, 30, 1906148.

26 A. Patil, Y. Ikenoue, N. Basescu, N. Colaneri, J. Chen, F. Wudl and A. Heeger, Self-doped conducting polymers, Synth. Met., 1987, 20, 151-159.

27 X. Chen and G. Chang, Synthesis and Photosensitive Properties of Poly (Aryl Imino) Containing Azobenzene Group (PAI-A), Chin. J. Chem., 2009, 27, 2093-2096.

28 C. Qin, X. Wu, H. Tong and L. Wang, High Solubility and Photoluminescence Quantum Yield Water-Soluble Polyfluorenes With Dendronized Amino Acid Side Chains: Synthesis, Photophysical, and Metal Ion Sensing Properties, J. Mater. Chem., 2010, 20, 7957-7964.

29 D. Bartholome and E. Klemm, Novel Polyarylene-Triarylmethane Dye Copolymers, Macromolecules, 2006, 39, 5646-5651.

$30 \mathrm{X}$. Guo and M. D. Watson, Conjugated Polymers From Napthalene Bisimide, Org. Lett., 2008, 10, 5333-5336.

31 C. K. Song, B. J. Eckstein, T. L. D. Tam, L. Trahey and T. J. Marks, Conjugated Polymer Energy Level Shifts in Lithium-Ion Battery Electrolytes, ACS Appl. Mater. Interfaces, 2014, 6, 19347-19354.

32 M. J. Frisch, G. W. Trucks, H. B. Schlegel, G. E. Scuseria, M. A. Robb, J. R. Cheeseman, G. Scalmani, V. Barone, G. A. Petersson, H. Nakatsuji, X. Li, M. Caricato, A. V. Marenich, J. Bloino, B. G. Janesko, R. Gomperts, B. Mennucci,
H. P. Hratchian, J. V. Ortiz, A. F. Izmaylov, J. L. Sonnenberg, D. Williams-Young, F. Ding, F. Lipparini, F. Egidi, J. Goings, B. Peng, A. Petrone, T. Henderson, D. Ranasinghe, V. G. Zakrzewski, J. Gao, N. Rega, G. Zheng, W. Liang, M. Hada, M. Ehara, K. Toyota, R. Fukuda, J. Hasegawa, M. Ishida, T. Nakajima, Y. Honda, O. Kitao, H. Nakai, T. Vreven, K. Throssell, J. A. Montgomery, Jr., J. E. Peralta, F. Ogliaro, M. J. Bearpark, J. J. Heyd, E. N. Brothers, K. N. Kudin, V. N. Staroverov, T. A. Keith, R. Kobayashi, J. Normand, K. Raghavachari, A. P. Rendell, J. C. Burant, S. S. Iyengar, J. Tomasi, M. Cossi, J. M. Millam, M. Klene, C. Adamo, R. Cammi, J. W. Ochterski, R. L. Martin, K. Morokuma, O. Farkas, J. B. Foresman and D. J. Fox, Gaussian16 Revision C.01, Gaussian Inc. Wallingford CT, 2016.

33 Handbook of Conducting Polymers, ed. J. R. Reynolds, B. C. Thompson and T. A. Skotheim, CRC Press, 4th edn, 2019, vol. 2.

34 C. C. Han and R. L. Elsenbaumer, Protonic Acids: Generally Applicable Dopants for Conducting Polymers, Synth. Met., 1989, 30, 123-131.

35 F. Wudl, R. O. Angus, Z. X. Liu, A. J. Heeger, F. L. Lu, P. M. Allemand, D. J. Vachon and M. Nowak, Poly( $p$-Phenyleneamineimine): Synthesis and Comparison to Polvaniline, J. Am. Chem. Soc., 1987, 109, 3677-3684. 